University of Nebraska - Lincoln

DigitalCommons@University of Nebraska - Lincoln

Faculty Publications in Food Science and Technology

Food Science and Technology Department

6-2011

\title{
The Human Gut Microbiome: Ecology and Recent Evolutionary Changes
}

Jens Walter

Ruth Ley

Follow this and additional works at: https://digitalcommons.unl.edu/foodsciefacpub

Part of the Food Science Commons, Genetics and Genomics Commons, and the Microbiology Commons

This Article is brought to you for free and open access by the Food Science and Technology Department at DigitalCommons@University of Nebraska - Lincoln. It has been accepted for inclusion in Faculty Publications in Food Science and Technology by an authorized administrator of DigitalCommons@University of Nebraska Lincoln. 
Annu. Rev. Microbiol. 2011. 65:411-29

First published online as a Review in Advance on June 16, 2011

The Annual Review of Microbiology is online at micro.annualreviews.org

This article's doi:

10.1146/annurev-micro-090110-102830

Copyright (c) 2011 by Annual Reviews. All rights reserved

0066-4227/11/1013-0411\$20.00

\section{The Human Gut Microbiome: Ecology and Recent Evolutionary Changes}

\author{
Jens Walter ${ }^{1}$ and Ruth Ley ${ }^{2}$ \\ ${ }^{1}$ Department of Food Science, University of Nebraska, Lincoln, Nebraska 68583-0919 \\ ${ }^{2}$ Department of Microbiology, Cornell University, Ithaca, New York 14853; \\ email: rel222@cornell.edu
}

\section{Keywords}

host-microbe interactions, ecological theory, niche, microbial diversity, human genetics, amylase

\begin{abstract}
The human gastrointestinal tract is divided into sections, allowing digestion and nutrient absorption in the proximal region to be separate from the vast microbial populations in the large intestine, thereby reducing conflict between host and microbes. In the distinct habitats of the gut, environmental filtering and competitive exclusion between microbes are the driving factors shaping microbial diversity, and stochastic factors during colonization history and in situ evolution are likely to introduce intersubject variability. Adaptive strategies of microbes with different niches are genomically encoded: Specialists have smaller genomes than generalists, and microbes with environmental reservoirs have large accessory genomes. A shift toward a Neolithic diet increased loads of simple carbohydrates and selected for their increased breakdown and absorption in the small intestine. Humans who outcompeted microbes for the new substrates obtained more energy from their diets and prospered, an evolutionary process reflected in modern population genetics. The three-way interactions between human genetics, diet, and the microbiota fundamentally shaped modern populations and continue to affect health globally.
\end{abstract}




\section{Contents}

INTRODUCTION ................ 412

GUT HABITATS AND THEIR

INHABITANTS .............. 412

Stomach and Small Intestine....... 414

Large Intestine .............. 414

MICROBIAL ECOLOGY

OF THE GUT ............... 415

Determinants of a Highly Individual Microbiome Composition ....... 416

Gut Niches................. 419

RECENT EVOLUTION OF THE

HUMAN-MICROBIOTA

SYMBIOSIS.

Changes to the Microbiome over

Human Evolution............ 422

CONCLUDING REMARKS ....... 423

\section{INTRODUCTION}

A fast and powerful way for an organism to take on new faculties is to acquire the necessary genes from another organism. In microbes, the

Niche: the

complement of activities that support the life of an organism, commonly compared to profession

Microbiota: microbial life forms within a given habitat or host

Metagenome: community-level genome composed of the combined genomes of its constituents

Habitat: the dwelling place of an organism

Microbiome: microbial life forms inhabiting a living host, their combined genomes, and their interactions with the host ecological expansion into new niches is often facilitated by the uptake of gene sequences from other microbes via horizontal gene transfer (91). In contrast, multicellular eukaryotes do not take up exogenous DNA as readily as microbes; instead, they form symbiotic associations with microbes that carry the necessary genes, allowing a rapid adaptive extension of their phenotypic capabilities (78). Hostmicrobe symbiosis is widely distributed within the Eukaryota, and the molecular mechanisms that underlie these relationships and the contributions made by the microbes toward their host's biology are well understood in certain invertebrates (e.g., insects and squids) $(57,64)$. Many of the interactions that have emerged between symbiotic partners are mutualistic and enhance the fitness of the host, and in insects, those include contribution to nutrition and defense.

Host-microbe symbiosis in humans and other mammals differs from that in inverte- brates because vertebrate hosts generally harbor more diverse and dense microbial communities in their intestines $(45,58)$. The human gut microbiota as a whole encode 150 times more genes in their collective metagenome than are present in the human host genome (70). Traits humans have gained from the acquisition of microbial symbionts include biosynthetic capacities to break down a greater range of plant polysaccharides; microbial fermentation provides roughly $10 \%$ of daily energy from a Western diet (8). But human gut microbes appear to have retained many genes also present in the host genome (70). Host enzymes that are active in food degradation have equivalents in most if not all gut bacterial genomes (51). This stands in contrast to the mutualisms of insects, in which either bacteria or host loses redundant functions and as a result they become irreversibly dependent on each other for nutrition (60).

In this review, we focus on the ecology and evolution of the human gut microbiota over the long term and in very recent human evolution. We discuss the current understanding of microbial biomass and diversity in the small and large intestines, the functions of microbes in these habitats, and the ecological forces that have shaped the gut microbiome. We present evidence that the community composition of the gut microbiota and the genetic content of bacterial genomes are determined largely by adaptations to niches whose characteristics are shaped by host genotype and microbial interactions within the communities. We then discuss how very recent culturally driven changes in diet are likely to have challenged the competitive balance of host and gut microbiota and to have affected the evolution of this symbiosis.

\section{GUT HABITATS AND THEIR INHABITANTS}

The human digestive tract is partitioned to segregate the host digestive processes from most of the microbial biomass, so that the host has the first shot at dietary substrates. Host digestive enzymes (e.g., amylase and lipase) are secreted from the salivary glands (Figure 1) 


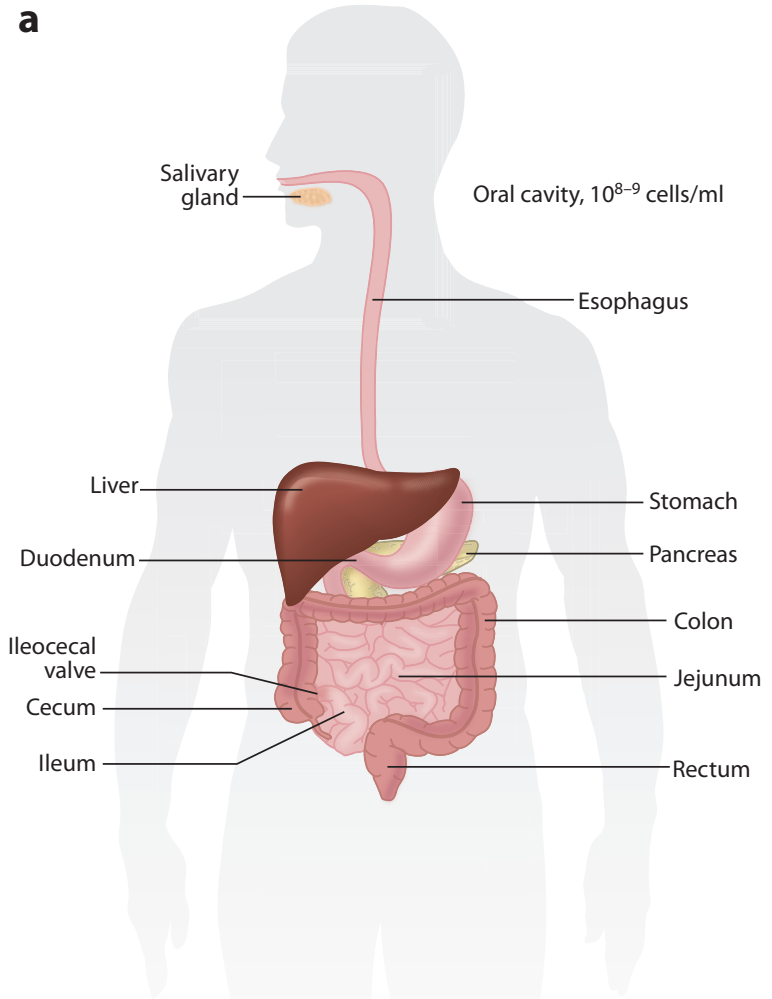

\section{Bacterial population present}

Oral cavity:

Gemella (e.g., G. haemolysans), Granulicatella, Streptococcus (e.g., S. mitis), Veillonella, Prevotella, Porphyromonas, Rothia, Neisseria, Fusobacterium Lactobacillus

Allochthonous microbes are generally outnumbered by autochthonous microbes.

\section{Stomach:}

Helicobacter pylori

Allochthonous: Gemella (e.g., G. haemolysans), Granulicatella, Streptococcus (e.g., S. mitis), Veillonella, Prevotella, Porphyromonas, Rothia, Neisseria, Fusobacterium, Lactobacillus b

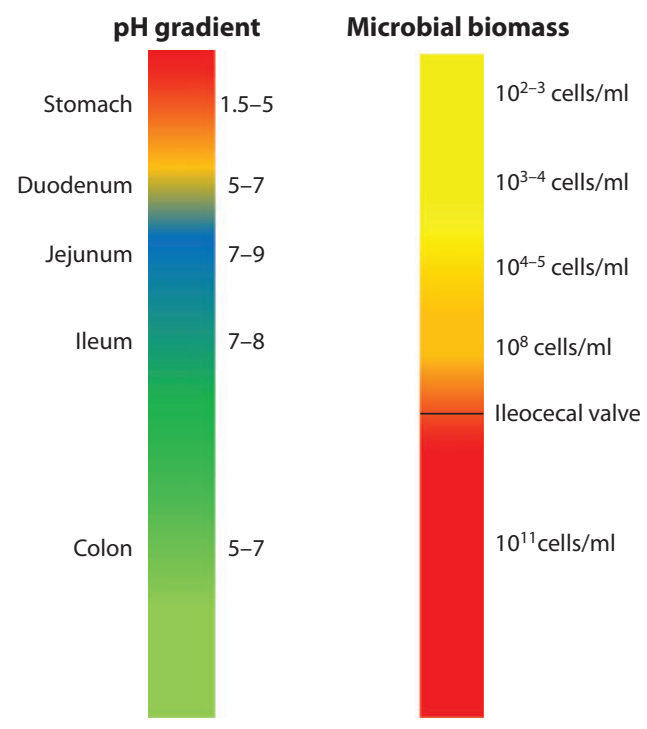

Small intestine:

Escherichia coli, Klebsiella, Enterococcus, Bacteroides, Ruminococcus, Dorea, Clostridium, Coprococcus, Weissella, Lactobacillus (some species)

Allochthonous: Granulicatella, Streptococcus (e.g., S. mitis), Veillonella, Lactobacillus

\section{Large intestine:}

Five major phyla: Firmicutes, Bacteroidetes, Actionobacteria, Verrucomicrobia, and Proteobacteria. Hundreds of species.

Allochthonous microbes are generally outnumbered by autochthonous microbes.

Figure 1

Characteristics of the major habitats of the human gastrointestinal tract and their inhabitants. (a) The major sections of the gastrointestinal tract. (b) Bars in the center indicate $\mathrm{pH}$ levels moving from the stomach to the distal gut (left) and biomass levels (right). (c) Boxes indicate the dominant types of microbes either allochthonous or autochthonous to those habitats. 
during mastication, and the stomach retains the food and provides the acidic $\mathrm{pH}$ for the host's proteases to be active. Additional enzymes (e.g., proteases, lipases, and amylase) are added from the pancreas and liver via the biliary ducts in the small intestine (SI). Food components that are degradable by the digestive enzymes are broken down to simple sugars, amino acids, and fatty acids, which are absorbed in the SI. Food components that escape digestion (fiber, resistant starch, some peptides and lipids) are passed via the ileocecal valve into the large intestine (LI). This valve is critical for host-commensal relations, as it limits the reflux of colonic contents into the ileum, restricting the vast majority of the biomass present in the human gastrointestinal tract to the LI. It is in the LI that the saccharolytic bacteria extract additional energy from the diet, including those nondigestible carbohydrates from plant and animal sources that are resistant to human enzymes (30).

\section{Stomach and Small Intestine}

The microbiota of the proximal gastrointestinal tract can contribute to the amino acid requirements of the host if they are not provided by the diet itself $(72,98)$. Despite these contributions, microbial biomass is kept relatively low in the proximal SI of humans: Cell densities reach levels of $10^{4}-10^{5}$ cells per milliliter of effluent, with higher populations $\left(10^{8}\right.$ cells per milliliter) in the ileum (Figure 1). The bacterial populations associated with the mucosa of the SI include the phylum Bacteroidetes and members of the Clostridiales clusters XIV and IV $(106,107)$, and those of the lumen can include members of the Enterobacteriaceae $(11,34)$ (Figure 1). Low biomass levels limit competition for substrates: The microbiota would certainly win over the host owing to their small surface-to-volume ratios and proximity to the substrates. Indeed, when bacterial biomass is too high in the SI, malabsorption of nutrients can occur (3).

There are several ways by which the human host restricts bacterial biomass in the stomach and SI. The low $\mathrm{pH}$ of gastric contents and rapid lumenal flow tend to limit microbial growth (40). In addition, bile salts, which are secreted into the duodenum via the bile, are strongly bactericidal. Another tool in the host arsenal for controlling bacteria is immunoglobulin (Ig). IgA recognizes the dominant microbes present (32). The majority of IgA is thought to be relatively unselective because it binds with epitopes that are widely shared among gut bacteria (89). IgA is generally thought to limit microbial penetration into the mucosa, and it can trigger bacterial agglutination in the mucus, enhancing clearance via peristalsis $(36,54)$. Although the mechanism involved is not well understood, IgA may also shape the diversity of the normal gut microbiota and reduce the amount of bacteria in the proximal SI (90).

In addition to IgA, the host produces antimicrobial compounds (e.g., defensins, cathelicidins, and C-type lectins) in all epithelial cell lineages $(12,36)$. Many are expressed constitutively (69). Others (e.g., C-type lectins) are triggered by the presence of bacteria (103). These compounds vary in their killing selectivity $(14,37)$. Altered levels of the expression of $\alpha$-defensins in transgenic animals have demonstrated their potential to alter the community composition of the lumenal microbiota, but not biomass levels (79). Naturally occurring levels of antimicrobial peptides are far lower in the lumen than in the mucosa (36), so their impact on microbial lumenal populations is uncertain.

\section{Large Intestine}

Several properties of the LI allow bacterial proliferation: less acidic $\mathrm{pH}$, larger volume, lower concentrations of bile salts, and longer retention time due to slower peristalsis. The LI lacks Peyer's patches present in the SI that sample the bacteria and direct immune responses. As a result, the host's immune system can tolerate densities of bacteria exceeding $10^{11}$ cells per gram content. Microbial activity in the colon produces substantial amounts of short-chain fatty acids (SCFAs) by fermentation of dietary compounds that escape digestion in the SI, and from endogenous substrates such as carbohydrates 
from shed mucus, proteins from host cells, and enzymes. SCFAs, particularly butyrate, provide most of the LI enterocyte energy requirements. However, the LI lacks the ability to actively absorb amino acids and B vitamins, so the host can gain from the presence of these compounds only if they are fermented to SCFAs, or if they support beneficial microbial growth (88).

Like the SI, the substantial majority of the mucosally associated populations of the LI belong to the Firmicutes (clusters IX, XIV, and $\mathrm{XVI}$ ) and Bacteroidetes (23) (Figure 1). Other phyla represented include the Actinobacteria, which can be common in the feces of some individuals (102), the Verrucomicrobia (i.e., Akkermansia, Victivallis), and a number of less abundant phyla such as the Proteobacteria and Fusobacteria (15). The phylogenetic architecture of the LI microbiota can be described as fan-like, with a high diversity of strains representing a few deep evolutionary lineages $(6,47)$. Unlike the high species/strain diversity of Bacteria seen in the LI, the Archaea are represented primarily by Methanobrevibacter smithii and, to a lesser degree, by Methanosphaera stadtmanae (23). In addition to Bacteria and Archaea, other types of microbes are present, such as protozoans and fungi (81), whose functions are less well understood. Viruses, mostly prophages and phages whose hosts are prominent bacterial members of the microbiota, are remarkably common in the human LI and probably play an important role in the evolution and ecology within the ecosystem (75). Standard biopsy samples show little variation in mucosal community diversity along the length of the colon, indicating a relatively uniform or well-mixed system $(23,111)$, and fecal microbiota represent a mix of mucosally associated and lumenal populations $(23,47)$.

\section{MICROBIAL ECOLOGY OF THE GUT}

The widespread use of molecular techniques has allowed greater insight into the composition of host-associated microbial communities and has revealed that the distinctive environ- mental characteristics along the gastrointestinal tract result in the development of specific populations that inhabit these habitats (Figure 1) $(10,11,15,23)$. However, most studies consist of single timepoint snapshots of diversity that cannot differentiate between permanent and transient members, and this distinction is particularly important for interpretation of temporal dynamic within these populations.

On the basis of ecological observations in mice, Savage (80) suggested that microbial communities in the gastrointestinal tract contain autochthonous and allochthonous members, and these categories provide a useful framework for understanding patterns of diversity and stability in the human gastrointestinal tract. Autochthonous members occupy physical spaces (niches) and form stable populations over long periods (80). In contrast, allochthonous microbes lack specific niches (i.e., profession), and although they may be found in any given habitat in significant numbers, they contribute little to the economy of the ecosystem (80). The microbial and viral communities found in human fecal samples are relatively stable over time $(15,48,75)$ and remarkably resistant to blooms of subpopulations, dietary changes (56), and antibiotics in moderate doses (77). These findings indicate that the microbial communities present in the LI are to a large degree dominated by autochthonous microbes.

Microbiota of body sites with low population densities, such as the SI, tend to be less stable over time $(11,34)$. One reason for the inverse correlation between stability and population size is that allochthonous microbes are more likely to be detected against a small autochthonous population than a large one. For example, from the hundreds of phylotypes detected in the human stomach (10), only Helicobacter pylori persists in this ecosystem (Figure 1), is absent from the oral cavity or food, and possesses the phenotypic and genomic traits to survive in the stomach (97). In contrast, many of the bacterial groups that are detectable by molecular methods in the stomach and SI are also dominant in the oral cavity, from which they likely originate
Autochthonous: endogenously derived and common to a majority of hosts

Allochthonous:

derived from outside sources, not found in the majority of hosts, but can be abundant in any one host 
(e.g., Streptococcus mitis, Veillonella spp., and Granulicatella spp.) $(1,10,11)$ (Figure 1). Thus, introducing high amounts of allochthonous microbes might contribute to the temporal variation in the SI (11). Despite its high temporal variation, the human SI is likely to still possess an autochthonous microbiota in addition to $H$. pylori, but identification of autochthonous residents will require repeated sampling over time and would benefit from a characterization of the genomic underpinnings of the persistent members (105).

\section{Determinants of a Highly Individual Microbiome Composition}

To be autochthonous to a particular host, microbes should not only stably colonize the gastrointestinal tract, but also be present in a majority of individuals (80). These characteristics certainly apply to the dominant bacterial phyla of the gut, and specific lineages have also been described in several studies as components of a phylogenetic core (e.g., taxa present in 50\% to $90 \%$ of individuals surveyed) $(70,92,108)$. However, the concept of a phylogenetic core is defined differently between studies, and only a small number of species-level lineages have ever been detected in all subjects surveyed (70), as the gut microbiota differ greatly between subjects in membership and community structure $(23,102)$. Despite differences in composition between individuals, microbiomes appear largely functionally equivalent (102). In this section we discuss how the patterns of microbial diversity within and between individuals can be explained with modern concepts of community ecology.

Humans are born with a sterile gastrointestinal tract that is successively colonized with microbial populations until adult-like communities stabilize (42). This process has been described as chaotic, owing to a lack of commonly shared temporal patterns of colonization in infants and an incomplete understanding about the events that shape patterns of colonization $(42,66)$. Current theory in community ecology proposes three processes that drive commu- nity assembly: (a) deterministic niche-related, (b) historic, and (c) neutral $(13,24)$. All three types of processes likely combine to shape the assembly of the gut microbiota, but the ecological features of the human gut microbiota suggest differences in their relative importance.

Niche-related, deterministic processes that follow definitive rules dictated by local environmental filters are undoubtedly an important shaper of gut microbial diversity $(13,24)$. In this view, communities within the gastrointestinal tract of individuals are composed of superior competitors that assemble themselves according to available niches. These processes select for members possessing specific traits, and community structure is to a large degree regulated by competitive exclusion $(33,41)$. The environment in this case is the host, which provides the physical and chemical properties of the habitat, thus imposing an initial gauntlet of selection (47). In response, gut bacteria possess traits in common, many of which allow homeostatic interactions with the host, such as the ability to vary surface polysaccharides $(43,68)$. Other traits enable persistence in the gut, such as the ability to degrade sialic acid via $\mathrm{N}$-acetylneuraminic lyases (2), and other components of host mucins, when preferred dietary substrates are depleted (85). Strong environmental filters in the gut that impose strict requirements for successful colonization are likely the main reasons why gut bacteria form lineages that are distinct from those of environmental microbes (46).

The importance of deterministic forces in the assembly of the gut microbiota has been demonstrated empirically: When microbial communities are transplanted from conventionally raised mice to germ-free zebrafish and vice versa, the community composition changes to resemble the normal gut microbial community of the recipient host (74). The shift in the relative abundances of microbes when placed in a different host shows that the outcome of competition between different subsets of a given microbiota is dependent on the environmental conditions presented by the different hosts. Even within a single host species, 
genetic differences between hosts can underlie differences in gut community composition. Host genetic factors, mostly relating to immunity, have a clear measurable contribution to the relative abundance of bacterial genera (7).

However, filtering by the host, and competitive interactions between members of the microbiota, can explain only a fraction of the variability observed between subjects (18). Using a quantitative genetics approach, Bensen and colleagues (7) showed that host genetic factors accounted for some of the variation between mouse gut microbiota, leaving a substantial fraction to be explained by other factors. Similarly, the microbiota of genetically identical human monozygotic twins can show a similar degree of variability when compared with dizygotic twin pairs (102). These findings further indicate that environmental and stochastic factors affect community composition.

A second important factor that influences community assembly and diversity is colonization history $(13,24)$. In this view, historical patterns of dispersal, which can only be described in probabilistic terms, influence the interactions within the community and have more impact on community composition than environmental filters do. Differences in immigration order in particular affect community assembly, as organisms alter niches for themselves and other organisms, thereby shaping the physicochemical properties of the habitat and species interactions. This historical perspective of community assembly also opens the door for the consideration of evolutionary processes in community ecology $(13,24)$ (Figure 2). This perspective challenges two assumptions of classical ecology: (a) The traits of the members within a community are static over ecological timescales, and (b) communities assemble mainly by ecological fitting in which new members that evolved elsewhere fit themselves into the community's open niches. Instead, both old residents and recent arrivals may occupy new niches that appear during community assembly, a process known as in situ evolution $(13,24)$. Bacteria have many unique features that favor rapid evolution over ecological timescales: vast populations, short generation times, rapid mutation rates, phenotypic plasticity, and high levels of gene flow. Adaptive radiation can be remarkably fast in bacterial populations (71), and in a complex microbial community such as the gut microbiota, horizontal gene transfer [which is especially prevalent in gut ecosystems (91)] allows rapid adaptation to environmental changes and novel opportunities during assembly. As a result, niches opening during assembly of the gut microbiota can be populated either by acquisition of preadapted species or by the evolution of adaptive phenotypes within species already present (24). In situ evolution may play a central role in the establishment of the human gut microbiota, although the extent to which this happens over the life of a host is not known.

An alternative perspective to those discussed above states that community assembly is largely a neutral process in which species are ecologically equivalent (39). In this model, the composition at a local scale (individual host) and $\beta$-diversity is shaped by dispersal limitation, disturbance, and stochastic processes; specialization for habitats does not play a role in shaping diversity (39). The neutral model for community assembly has been tested for several microbial habitats, including the human gut, and although the model performs well for many microbial communities, it does not appear to predict community composition in human fecal samples (82). In addition, ecological communities assembled by neutral processes are open to additional colonists, are continuously changing, and have nonequilibrium assemblages of species $(13,24)$. This is in clear contrast to microbial communities in the LI, which are temporally stable, resilient to perturbations, and resistant to colonization $(15,19,56,87)$.

Last, many characteristics of gut microbiota can be explained when we assume a combination of niche-related and historical processes during community assembly. For instance, unpredictable events during assembly would result in differences in community structure in individuals ( $\beta$-diversity), whereas in situ evolution would ensure highly adapted phenotypes even in the absence of reliable modes of transmission 
a
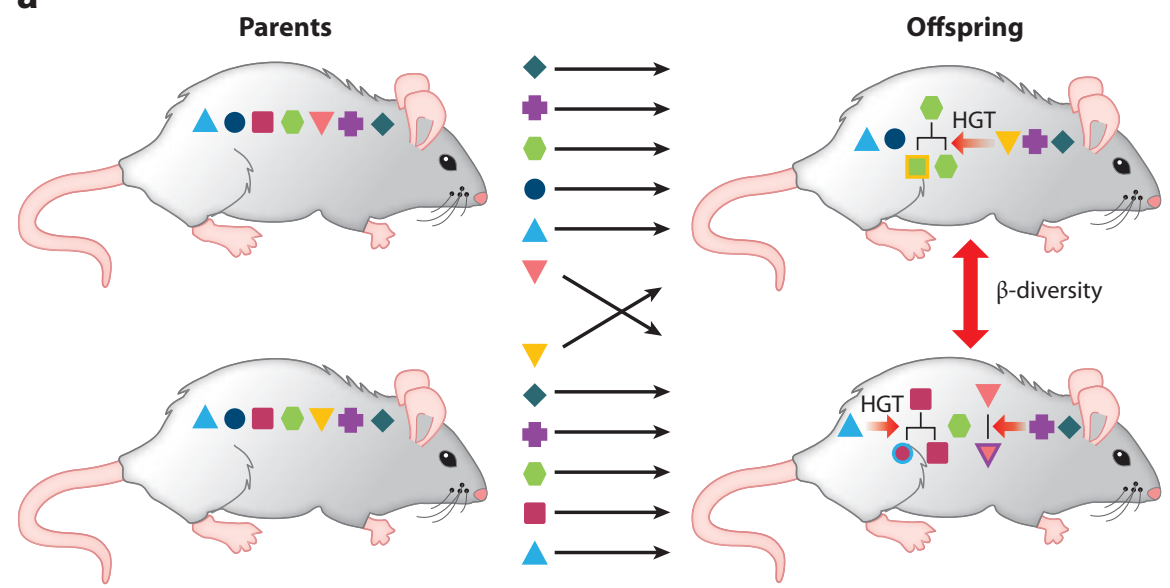

b
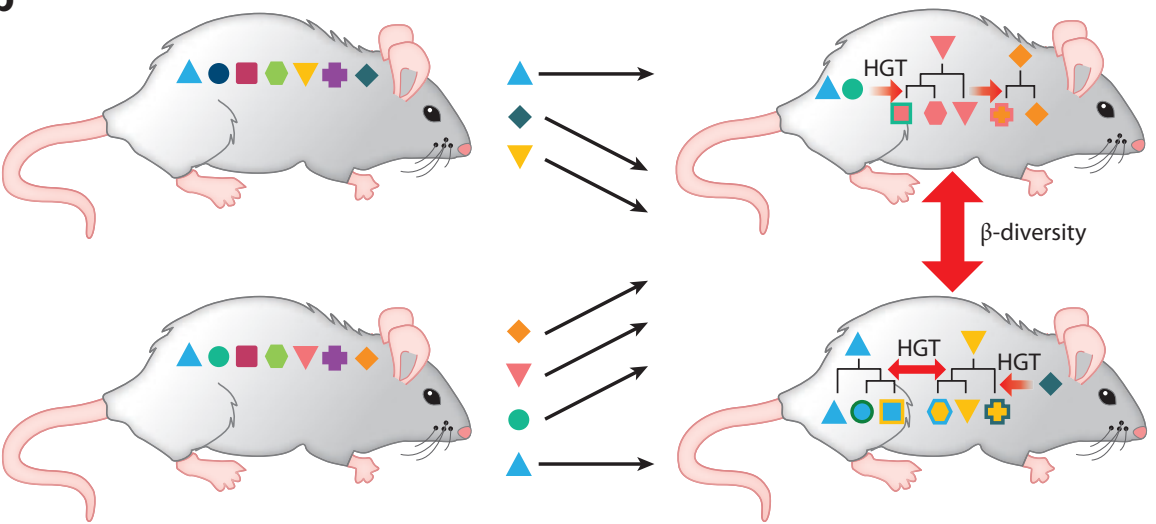

Figure 2

Model of the acquisition, in situ evolution, and community assembly of the gut microbiota. Transmission of microbes is indicated by arrows. Two extreme scenarios are shown. (a) Efficient vertical transmission from parent to offspring: Niches fill quickly by colonization of microbes that are preadapted. Adaptation to physiological differences in the niche environment of individual animals is facilitated by in situ evolution of early colonizers. Variation between individuals ( $\beta$-diversity) is due mostly to genetic differences within the population. (b) Inefficient vertical transmission from parent to offspring: Colonization of the next generation relies on horizontal transmission or dispersal. Immigration is slow, and in situ evolution/adaptation of early colonizers can occur more rapidly than colonization and becomes a main mechanism for filling available niche space. Variation between individuals is due mostly to stochastic and unpredictable environmental factors. Shapes indicate niches and colors indicate microbial lineages. Red arrows indicate gene flow. Abbreviation: HGT, horizontal gene transfer.

(Figure 2). Gene flow within members of the community would lead to phenotypic plasticity and functional redundancy among microbial lineages, with the result that microbiomes in adult humans are variable in composition but conserved in functional traits (102). Although these hypotheses seem to fit observational data, systematic experimental studies to test them are currently lacking. However, the relative importance of some interactions (e.g., 
competition and environmental filtering) can be inferred from the phylogenetic structure of communities $(13,38)$. Competition is thought to select for community members that are more distantly related to each other than predicted by random sampling of inoculum community (phylogenetic overdispersion), whereas host selection favors members more closely related than predicted (phylogenetic clustering) (24, 77). 16S rRNA gene-sequence-based studies show patterns of phylogenetic clustering: Microbial communities inhabiting the LI are rich in species and strain diversity belonging to relatively few phyla. This contrasts sharply with habitats, such as soil and seawater, that have a more even distribution of a wide number of phyla (46).

\section{Gut Niches}

The organisms that dominate the human LI vary tremendously in genome size and content (Table 1), reflecting differences in the niche characteristics and in the ecological and evolutionary strategies used by gut microbes to occupy these niches. Generally, organisms with larger genomes follow more generalist lifestyles. An example is Bacteroides thetaiotaomicron, which has evolved to utilize complex and dynamic nutrient sources. In contrast, organisms with smaller genomes, such as H. pylori and Lactobacillus reuteri, appear to be adapted toward a restricted menu of dietary substrates, and these microbes also show specificity toward particular host species.

The extreme conditions in the mammalian stomach and SI select for specialized microbes. H. pylori isolates from humans and Lactobacillus spp. from rodents and pigs can tolerate the acidic conditions present in the stomach of their hosts $(28,97)$. Enterobacteria (e.g., Escherichia coli) and Enterococcus spp., which are commonly found in the human SI (34), can tolerate high concentrations of bile acids. $H$. pylori, lactobacilli, enterobacteria, and enterococci have limited abilities to utilize complex carbohydrates: Their nutritional requirements reflect the nutrient-rich habitats they occupy, which are proximal to host-nutrient absorption.

In addition to being specialized to a specific gut habitat, species within the genus Helicobacter are remarkably specific for particular hosts (83). For example, $H$. pylori is associated with humans (49), and $H$. hepaticus is associated with mice. Gastric Helicobacter species are autochthonous: They have been (until the antibiotic age) nearly universally prevalent and usually not pathogenic to their natural host. Another example of a highly host-adapted gut microbe is $L$. reuteri $(28,65)$. The population

Table 1 Genome sizes, habitats, and niches of human gut bacteria

\begin{tabular}{l|l|l|c}
\hline Organism & \multicolumn{1}{|c|}{ Habitat } & \multicolumn{1}{c}{ Ecological niche } & Genome size (Mb) \\
\hline Bacteroides thetaiotaomicron & Large intestine & Saccharolysis & 6.3 \\
\hline Bacteroides vulgatus & Large intestine & Saccharolysis & 5.2 \\
\hline Parabacteroides distasonis & Large intestine & Saccharolysis & 4.8 \\
\hline Escherichia coli & Small, large intestine & Fermentation of simple sugars and amino acids & 4.5 \\
\hline Roseburia intestinalis & Large intestine & Saccharolysis, butyrate producer & 4.2 \\
\hline Eubacterium rectale & Large intestine & Saccharolysis, butyrate producer & 3.4 \\
\hline Faecalibacterium prausnitzii & Large intestine & Saccharolysis, butyrate producer & 3.1 \\
\hline Bifidobacterium adolescentis & Large intestine & Dietary carbohydrates & 2.1 \\
\hline Methanobrevibacter smithii & Large intestine & Methanogen & 1.9 \\
\hline Lactobacillus reuteri & Small intestine & Host specific, fermentation of simple sugars and & \\
\hline Helicobacter pylori & Stomach & Utilization of simple sugars and amino acids & 2.0 \\
\hline
\end{tabular}


structure of $L$. reuteri indicates a long-term association of specific clades with particular hosts and host-driven diversification (65). However, many other microbial lineages follow a promiscuous lifestyle, because they are found in different host species (45). An example is $E$. coli, whose population structure is characterized by distinct clades that show no clear association with particular host species (94). The evolutionary strategy of $E$. coli appears to resemble that of facultative symbionts of insects, which are often erratically distributed and resemble invasive pathogens in that they spread through various host lineages (60).

The lifestyle and ecological roles of microbes affect the genome evolution and content of gut microbes. In an analysis of 20 commensal and pathogenic E. coli strains, Touchon et al. (99) noted more than 4,700 genes per strain. Of these, approximately 2,000 were estimated to be present in all E. coli strains; the total gene pool exceeded 10,000 genes (99). The ability of E. coli to survive outside the host is likely a selection pressure on par with host specialization and leads to expanded pan-genomes. A narrow host range has the opposite effect-genomes shrink. The reduced genome size of Helicobacter species and $L$. reuteri is reminiscent of other host-restricted bacteria $(61,63)$. Even though the genomes of $H$. pylori and L. reuteri, like E. coli, include genes acquired by horizontal gene transfer and form pan-genomes $(27,28)$, their genomes are nevertheless significantly smaller (Table 1) (99). In contrast to E. coli, both species are directly transmitted between hosts and lack environmental reservoirs. The genomic patterns observed could indicate an intermediate transitioning from a facultatively to an obligately host-associated lifestyle (28).

The numerically abundant saccharolytic bacteria of the human gut appear to be generalists in terms of niche preference. Their glycobiomes allow them to break down a great variety of polysaccharides. For instance, the genome of $B$. thetaiotaomicron contains 261 genes annotated in the CAZy database as encoding known or predicted glycoside hydrolases and polysaccharide lyases (55).
B. thetaiotaomicron can swiftly alter gene expression to match the ever-changing availability of its preferred growth substrates, so that the order of substrate preference, in addition to the potential to degrade a given substrate, further refines its niche specificity (85). Although capable of using a wide array of substrates, many microbes in the human LI also show specialization toward specific substrates. Genomically encoded specialization for specific glycans, such as inulin, can predict the competitive outcome between different species of Bacteroides (84). Bacteria such as Bifidobacterium adolescentis, Ruminococcus bromii, Eubacterium rectale, and Roseburia spp. expand their population sizes when specific dietary carbohydrates become available $(16,50,56,104)$.

If past competition between related strains of bacteria for substrates drives niche specialization and supports diversity, then a wider variety of glycans in the diet will lead to additional microbial niches and species in the gut. Indeed, herbivores have on average a more phylogenetically rich microbiota than carnivores do, reflecting their more complex dietary substrates (45). The diversity of the present-day human distal gut microbiota was assembled in response to our past diet: Preservation of high levels of diversity may require preservation of a varied diet rich in complex glycans.

\section{RECENT EVOLUTION OF THE HUMAN-MICROBIOTA SYMBIOSIS}

The development of agriculture and the domestication of animals have been major factors in recent human evolution $(20,76)$. Very early hominids were largely omnivorous and subsisted during periods of food scarcity on starch-rich roots and bulbs, especially in savannahs where edible plants were scarce (44). In the past 13,000 years, food production arose independently in several areas of the world, and diets were broadened to include high-starch plant foods and dairy products (20). We suggest that the subsequent changes in diet set the stage for intense conflict with diet substrates between 
host and gut microbiota. Host-microbiota conflict led to the selection of hosts that outcompeted microbes for simple carbohydrates, and possibly also reduced the microbial populations in the SI that competed for a refined diet containing additional starch and milk.

Starch is a complex polysaccharide consisting of a mixture of amylose (1,4- $\alpha$-linked glucose residues) and amylopectin, a branched polymer composed of amylose chains linked to an amylose backbone by 1,6- $\alpha$-linkages. The relative proportions of amylose and amylopectin in a diet item determine how well both the host and the bacteria can access it, and cooking foods can affect the properties of starch present in the diet (25). Digestion of starch begins in the mouth, where the activity of salivary $\alpha$-amylase contributes up to $\sim 40 \%$ of simple sugars (glucose) and short chains of partly digested starches (dextrins) (110). Once the food passes into the SI, pancreatic amylase continues the degradation process. Although similar, salivary and pancreatic amylases are encoded by different genes ( $A M Y 1$ and $A M Y 2$, respectively) and show different levels of activity against starches of different origin (31).

The genetics of modern human populations suggest that selection favored humans that could more effectively degrade starch in the mouth. $A M Y 1$ gene copy number in the human genome is related positively to levels of amylase in the saliva (67) and to oral enzymatic activity (53). People from traditionally agricultural areas, such as Europe and part of Asia, have on average a greater $A M Y 1$ copy number than people with hunter-gatherer backgrounds (67). This is one of the most dramatic signs of recent human evolution yet detected.

If starch intake exceeds the host's capacity to degrade it and absorb breakdown products during digestion, it will pass to the colon, where bacteria will ferment it. Gut microbes can degrade starch: Amylases are widely shared among phylogenetically unrelated gut microbes, such as Butyrivibrio, Roseburia, and Bacteroides (73). In Bacteria, amylase activity requires just three genes of the same family, whereas the degradation of $\beta$-glucans and xylans can require up to $17 \mathrm{CAZyme}$ modules corresponding to 13 different CAZyme families (93). An increase in starch content in the diet can be met by populations of starch-utilizing bacteria, as these can expand in size in response to diet change (56). If bacteria in the LI could ferment the excess starch, why did selection favor individuals with high levels of salivary amylase production? The most likely answer is that the energy gains from direct uptake of starch monomers in the SI, instead of fermentation products from the LI, provided a fitness benefit to the host. In terms of straight energy content upon combustion, glucose $\left(2,890 \mathrm{~kJ} \mathrm{~mol}^{-1}\right)$ that is absorbed in the SI yields more energy than the SCFA (e.g., $2,144 \mathrm{~kJ} \mathrm{~mol}^{-1}$ for butyrate and $1,734 \mathrm{~kJ} \mathrm{~mol}^{-1}$ for 2 moles of acetate) (Table 2) that would be produced in the colon. Furthermore, the microbiota will divert some of the energy from the diet for their own maintenance and growth.

Table 2 Comparison of the energy released from combustion with oxygen at $1 \mathrm{~atm}$ and $37^{\circ} \mathrm{C}$ of glucose and its fermentation products ${ }^{\mathrm{a}}$

\begin{tabular}{l|c|c|c}
\hline Chemical & Number of electrons & Number of carbon atoms & $\mathbf{k J ~ m o l}^{\mathbf{- 1}}$ \\
\hline Glucose & 24 & 6 & 2,890 \\
\hline Butyrate & 20 & 4 & 2,144 \\
\hline Propionate & 13 & 3 & 1,502 \\
\hline Lactate & 12 & 3 & 1,361 \\
\hline Ethanol & 12 & 2 & 1,319 \\
\hline Acetate & 8 & 2 & 867 \\
\hline
\end{tabular}

${ }^{\mathrm{a}}$ Data from Reference 4. 
These energy-yield considerations imply that the optimal strategy for a host evolving with a high starch diet was breakdown and uptake of simple sugars in the SI, with minimal enzymatic contribution from bacteria in the LI.

Milk was also incorporated into the diet during the Neolithic transition to a pastoral life. All nonhuman mammals lose their ability to degrade lactose soon after weaning owing to decreased levels of lactase-phlorizin hydrolase in the gut. The lactase persistence (LP) trait in humans is a dominant Mendelian trait common in places with a long history of raising animals for milk production. There are several ways that human genetics have changed to achieve LP, which is an example of convergent evolution of this phenotype in human populations (95). Human genetic evidence indicates that a selective sweep occurred in European populations between 5,000 and 10,000 years ago (9). Lactose that is not assimilated in the SI passes into the colon to be fermented by bacteria. Many gut bacteria have $\beta$-galactosidases, which break down lactose into glucose and galactose. As with starch, the positive selection among milk-consuming peoples for LP suggests a larger energetic gain compared with allowing lactose fermentation in the gut. Clearly, lactose and starch have similar histories and effects on human populations. When added to the diet, selection favored humans who utilized both substrates in the SI more efficiently. The microbiome was capable of degrading these compounds (niches were occupied), but hosts who maximized these capabilities independently of bacteria had the greatest fitness.

In addition to selection for specific host genotypes, it is tempting to speculate that another host response to Neolithic diet changes was a restriction of microbial densities in the SI. Animals such as mice, rats, pigs, and horses harbor much higher microbial populations in their proximal intestinal tract. The relatively strict spatial compartmentalization of digestion between host and microbe in modern humans might be a direct evolutionary response to the increase in easy digestible diets during the
Neolithic revolution. This would have allowed humans to enjoy their refined diets without microbial competition while still benefiting from the fermentation of fiber and resistant starches in the colon.

\section{Changes to the Microbiome over Human Evolution}

Direct evidence for changes in the microbiota over recent human history is almost impossible to come by because well-preserved intestinal or fecal specimens are extremely rare. One notable metagenomic analysis of ancient feces (1,300 years old, Mexico) revealed Methanobrevibacter smithii as the only archaeon present, a dominance of Bacteroidetes and Firmicutes, and major functional categories, such as carbohydrate metabolism and metabolism of vitamins, similar to features seen in modern microbiomes $(70,96)$.

Going back farther in time, one early change in hominid diet came with the invention of cooking, which most likely preceded agriculture. Cooking would have allowed early humans to consume a wider range of foods that were otherwise too tough for human consumption $(21,52)$. Great apes today prefer cooked tubers and beef over raw options, suggesting that early hominids might have started eating cooked foods as soon as fire was under control (109). Cooking may have shaped the gene content of the human microbiome by introducing new toxins (i.e., the Maillard reaction produces acrylamide) $(62,86)$, and gut bacteria can further transform products of the Maillard reaction (100). The presence of xenobiotics unique to cooked food may explain why some of the human gut microbiome's enzymes that degrade xenobiotics, such as unique $\beta$-glucuronidases, appear to be unique to the human gut (29).

We know that the microbiome adapts to changes in human ecology over time. A classic example is the biogeographic patterns of $H$. pylori that mirror those of human migration $(26,59)$. H. pylori has been passed between close relatives over generations, such that its 
strain variation can be mapped onto modern populations to reconstruct their histories (5). To date, data are insufficient to determine whether patterns of overall gut microbial community diversity also reflect human migration. But the generalist lifestyle of the majority of gut microbes, as described above, makes this less likely. The variation in human diet across the world can also confound patterns of gut microbial diversity. Diet may be selecting on existing differences in microbial populations that codiversified with human populations. A recent study of rural children in Burkina Faso and Italy showed that the Bacteroidetes were far more abundant in the African children's microbiomes (17). In addition, the types of Bacteroidetes present in the African children's microbiomes differed from those in typical Western or Asian microbiomes as they may be ideally suited to liberate energy from plant-rich diets typical of the African children. Microbiomes vary geographically with their hosts in part because they are adapted to local diets.

Adaptation to local diet can occur by acquisition of novel genes by the resident microbiota. A spectacular example of this has been shown for Bacteroides plebeius. Strains of B. plebeius isolated from Japanese subjects harbor a gene acquired from marine bacteria and necessary to degrade porphyran in edible seaweed (nori), and this gene is undetected in North American microbiomes (35). Particularly significant was the demonstration that a local adaptation could be achieved by incorporating genes from the environment in what constitutes clear evidence of past in situ evolution.

Within modern human populations, recent diet changes have undoubtedly affected the relative abundances of the types of microbes present in the gut (104). A Western diet low in complex carbohydrates and high in simple sugars and fat is associated with reduced levels of Bacteroidetes in North Americans (48, 101). Specific types of bacteria that are detectable in feces show increases in numbers in response to short-term dietary changes, such as changes in resistant starch content $(22,56,104)$. The gen- eral pattern over time is likely to be associated with a loss of microbial diversity as diets become more refined and antibiotics that are effective against autochthonous members, such as H. pylori and L. reuteri, are deployed.

\section{CONCLUDING REMARKS}

Humans maintain intimate associations with an autochthonous gut microbiota in a mutualism that has evolved over millennia. If the human microbiota are composed of lineages that form specialized mutualistic relationships with their host, then the acquisition of these microbes and the correct assembly of the microbiota early in life would be important for the development and lifelong health of the host. The assembly of the microbiota is a complex process that is to a large degree dependent on the transmission history of the microbes. It is almost inevitable that the characteristics of the modern lifestyle, such as antibiotics, Caesarian sections, hygiene, refined diets, formula feeding, and small households, would introduce hurdles into symbiont transmission with consequences for the functional development of the gut microbiota. This is one way in which the modern lifestyle might contribute to the rise in chronic diseases recently associated with the gut microbiome in westernized societies.

Our most recent evolution was driven in large part by changes in diet. We may arguably be seeing a new wave of intense changes in diet in subsets of the population, toward loss of fiber and greater intake of simple sugars, fats, and proteins. These changes might be driving not only the obesity epidemic, which has been associated with changes in microbial ecology of the gut (48), but also compositional and functional differences within the gut microbiota that predispose humans to metabolic disorders. One treatment of metabolic disease today is to inhibit amylase activity. It is ironic that one way to treat problems brought about by our modern diet is in effect to negate the consequence of gene duplications that allowed many of our ancestors to survive. 


\section{SUMMARY POINTS}

1. The human intestine is divided into discrete sections that spatially segregate host digestive activity in the stomach and SI, where microbial biomass is low, from high microbial biomass and enzymatic activity in the LI. The separation is necessary because most gut microbes possess the capacities of the host to degrade and take up simple substrates that are the main component of modern diet, but the host relies on additional properties of gut microbes to generate SCFAs.

2. The distinct regions of the gut create unique habitats to which the resident microbiota are well adapted. Within the constraints of the gut habitats, competition for niche space shapes microbial diversity. During assembly of the gut microbiota, stochastic events and adaptive processes further combine to influence the in situ evolution of gut microbiota and resulting patterns of diversity.

3. Autochthonous members are the most highly specialized to gut niches and are rarely found outside the gut. Those with restricted host ranges also have reduced genome sizes. The dominant bacteria of the colon are nutritional generalists able to degrade a wide variety of glycan substrates and divide niche space with different orders of substrate preferences. Little is currently known about the degree to which mammalian gut microbes show host specialization.

4. The development of agriculture altered human diets. Starch and milk were added to diets in many parts of the world. Human genetic evidence suggests that people able to utilize starch and milk directly using host enzymes, while minimizing microbial fermentation, had a fitness advantage.

5. Modern diets, which are even richer in simple substrates compared with Neolithic diets, together with features of the modern lifestyle, further stress the interactions between the microbiota and the human host, which might contribute to the global epidemic of metabolic disorders today.

\section{FUTURE ISSUES}

1. A more refined understanding of how the microbiota of the gut is assembled is necessary to inform strategies to reshape microbiota. Do successional stages exist and affect the nature of later-stage microbiota?

2. How rapidly does in situ evolution occur in the human gut, and does this process contribute substantially to the variability between subjects?

3. The microbiota of the SI are understudied compared to fecal microbiota, yet they interact closely with the host immune system. Research into their ecology and role in diseases is essential.

\section{DISCLOSURE STATEMENT}

The authors are not aware of any affiliations, memberships, funding, or financial holdings that might be perceived as affecting the objectivity of this review. 


\section{ACKNOWLEDGMENTS}

We thank Lars Angenent and Matt Agler for insights into microbial thermodynamics. Support was provided by The David and Lucile Packard Foundation, The Hartwell Foundation, and a Beckman Young Investigator award to REL.

\section{LITERATURE CITED}

1. Aas JA, Paster BJ, Stokes LN, Olsen I, Dewhirst FE. 2005. Defining the normal bacterial flora of the oral cavity. 7. Clin. Microbiol. 43:5721-32

2. Almagro-Moreno S, Boyd EF. 2009. Insights into the evolution of sialic acid catabolism among bacteria. BMC Evol. Biol. 9:118

3. Almeida J, Kim R, Stoita A, McIver C, Kurtovic J, Riordan S. 2008. Lactose malabsorption in the elderly: role of small intestinal bacterial overgrowth. Scand. F. Gastro. 43:146-54

4. AmendJ, Shock E. 2001. Energetics of overall metabolic reactions of thermophilic and hyperthermophilic Archaea and Bacteria. FEMS Microbiol. Rev. 25:175-243

5. Aspholm-Hurtig M, Dailide G, Lahmann M, Kalia A, Ilver D, et al. 2004. Functional adaptation of BabA, the $H$. pylori $\mathrm{ABO}$ blood group antigen binding adhesin. Science 305:519-22

6. Backhed F, Ley RE, Sonnenburg JL, Peterson DA, Gordon JI. 2005. Host-bacterial mutualism in the human intestine. Science 307:1915-20

7. Benson AK, Kelly SA, Legge R, Ma F, Low SJ, et al. 2010. Individuality in gut microbiota composition is a complex polygenic trait shaped by multiple environmental and host genetic factors. Proc. Natl. Acad. Sci. USA 107:18933-38

8. Bergman E. 1990. Energy contributions of volatile fatty acids from the gastrointestinal tract in various species. Physiol. Rev. 70:567-90

9. Bersaglieri T, Sabeti P, Patterson N, Vanderploeg T, Schaffner S, et al. 2004. Genetic signatures of strong recent positive selection at the lactase gene. Am. 7. Hum. Genetics 74:1111-20

10. Bik EM, Eckburg PB, Gill SR, Nelson KE, Purdom EA, et al. 2006. Molecular analysis of the bacterial microbiota in the human stomach. Proc. Natl. Acad. Sci. USA 103:732-37

11. Booijink CC, El-Aidy S, Rajilić-Stojanović M, Heilig HG, Troost FJ, et al. 2010. High temporal and inter-individual variation detected in the human ileal microbiota. Environ. Microbiol. 12:3213-27

12. Cash HL, Whitham CV, Behrendt CL, Hooper LV. 2006. Symbiotic bacteria direct expression of an intestinal bactericidal lectin. Science 313:1126-30

13. Cavender-Bares J, Kozak KH, Fine PV, Kembel SW. 2009. The merging of community ecology and phylogenetic biology. Ecol. Lett. 12:693-715

14. Cederlund A, Agerberth B, Bergman P. 2010. Specificity in killing pathogens is mediated by distinct repertoires of human neutrophil peptides. F. Innate Immun. 2:508-21

15. Costello EK, Lauber CL, Hamady M, Fierer N, Gordon JI, Knight R. 2009. Bacterial community variation in human body habitats across space and time. Science 326:1694-97

16. Davis LM, Martinez I, Walter J, Hutkins R. 2010. A dose dependent impact of prebiotic galactooligosaccharides on the intestinal microbiota of healthy adults. Int. F. Food Microbiol. 144:285-92

17. De Filippo C, Cavalieri D, Di Paola M, Ramazzotti M, Poullet JB, et al. 2010. Impact of diet in shaping gut microbiota revealed by a comparative study in children from Europe and rural Africa. Proc. Natl. Acad. Sci. USA 107:14691-96

18. Dethlefsen L, Eckburg PB, Bik EM, Relman DA. 2006. Assembly of the human intestinal microbiota. Trends Ecol. Evol. 21:517-23

19. Dethlefsen L, Huse S, Sogin ML, Relman DA. 2008. The pervasive effects of an antibiotic on the human gut microbiota, as revealed by deep 16S rRNA sequencing. PLoS Biol. 6:e280

20. Diamond J. 2002. Evolution, consequences and future of plant and animal domestication. Nature 418:700-7

21. Dominy N, Vogel E, YeakelJ, Constantino P, Lucas P. 2008. Mechanical properties of plant underground storage organs and implications for dietary models of early hominins. Evol. Biol. 35:159-75
7. The first use of principles of quantitative genetics to link variation in the host genome to variation in the gut microbiome. 
32. A clever system of reversible colonization reveals the memory dynamics of IgA.

35. Provides remarkable evidence for gene acquisition by a gut microbe as an adaptation to a local diet.
22. Duncan SH, Belenguer A, Holtrop G, Johnstone AM, Flint HJ, Lobley GE. 2007. Reduced dietary intake of carbohydrates by obese subjects results in decreased concentrations of butyrate and butyrateproducing bacteria in feces. Appl. Environ. Microbiol. 73:1073-78

23. Eckburg PB, Bik EM, Bernstein CN, Purdom E, Dethlefsen L, Sargent M. 2005. Diversity of the human intestinal microbial flora. Science 308:1635-38

24. Emerson BC, Gillespie RG. 2008. Phylogenetic analysis of community assembly and structure over space and time. Trends Ecol. Evol. 23:619-30

25. Englyst H, Kingman S, Cummings J. 1992. Classification and measurement of nutritionally important starch fractions. Euro. 7. Clin. Nutr. 46:S33-50

26. Falush D, Wirth T, Linz B, Pritchard JK, Stephens M, et al. 2003. Traces of human migrations in Helicobacter pylori populations. Science 299:1582-85

27. Fischer W, Windhager L, Rohrer S, Zeiller M, Karnholz A, et al. 2010. Strain-specific genes of Helicobacter pylori: genome evolution driven by a novel type IV secretion system and genomic island transfer. Nucleic Acids Res. 38:6089-101

28. Frese SA, Benson AK, Tannock GW, Loach DM, Kim J, et al. 2011. The evolution of host specialization in the vertebrate gut symbiont Lactobacillus reuteri. PLoS Genet. 7(2):e1001314

29. Gloux K, Berteau O, El Oumami H, Béguet F, Leclerc M, Doré J. 2011. A metagenomic $\beta$-glucuronidase uncovers a core adaptive function of the human intestinal microbiome. Proc. Natl. Acad. Sci. USA. 108:4539-46

30. Grabitske HA, Slavin JL. 2008. Low-digestible carbohydrates in practice. 7. Am. Dietetic Assoc. 108:167781

31. Hall F, Ratliff C, Hayakawa T, Culp T, Hightower N. 1970. Substrate differentiation of human pancreatic and salivary alpha-amylases. Am. F. Dig. Dis. Sci. 15:1031-38

32. Hapfelmeier S, Lawson MAE, Slack E, Kirundi JK, Stoel M, et al. 2010. Reversible microbial colonization of germ-free mice reveals the dynamics of IgA immune responses. Science 328:17059

33. Hardin G. 1960. The competitive exclusion principle. Science 131:1292-97

34. Hayashi H, Takahashi R, Nishi T, Sakamoto M, Benno Y.2005. Molecular analysis of jejunal, ileal, caecal and recto-sigmoidal human colonic microbiota using 16S rRNA gene libraries and terminal restriction fragment length polymorphism. 7. Med. Microbiol. 54:1093-101

35. Hehemann J, Correc G, Barbeyron T, Helbert W, Czjzek M, Michel G. 2010. Transfer of carbohydrate-active enzymes from marine bacteria to Japanese gut microbiota. Nature 464:90812

36. Hooper L, Macpherson A. 2010. Immune adaptations that maintain homeostasis with the intestinal microbiota. Nat. Rev. Immunol. 10:159-69

37. Hooper L, Stappenbeck T, Hong C, Gordon J. 2003. Angiogenins: a new class of microbicidal proteins involved in innate immunity. Nat. Immunol. 4:269-73

38. Horner-Devine MC, Bohannan BJ. 2006. Phylogenetic clustering and overdispersion in bacterial communities. Ecology 87:S100-8

39. Hubbell S. 2001. The Unified Neutral Theory of Biodiversity and Biogeography. Princeton, NJ: Princeton Univ. Press

40. Kanno T, Matsuki T, Oka M, Utsunomiya H, Inada K, et al. 2009. Gastric acid reduction leads to an alteration in lower intestinal microflora. Biochem. Biophys. Res. Commun. 381:666-70

41. Kassen R, Rainey PB. 2004. The ecology and genetics of microbial diversity. Annu. Rev. Microbiol. 58:207-31

42. Koenig J, Spor A, Scalfone N, Fricker A, Stombaugh J, et al. 2011. Succession of microbial consortia in the developing infant gut microbiome. Proc. Natl. Acad. Sci. USA 108:4578-85

43. Krinos CM, Coyne MJ, Weinacht KG, Tzianabos AO, Kasper DL, Comstock LE. 2001. Extensive surface diversity of a commensal microorganism by multiple DNA inversions. Nature 414:555-58

44. Laden G, Wrangham R. 2005. The rise of the hominids as an adaptive shift in fallback foods: plant underground storage organs (USOs) and australopith origins. F. Human Evol. 49:482-98

45. Ley RE, Hamady M, Lozupone C, Turnbaugh PJ, Ramey RR, et al. 2008. Evolution of mammals and their gut microbes. Science 320:1647-51 
46. Ley RE, Lozupone CA, Hamady M, Knight R, Gordon JI. 2008. Worlds within worlds: evolution of the vertebrate gut microbiota. Nat. Rev. Microbiol. 6:776-88

47. Ley RE, Peterson DA, Gordon JI. 2006. Ecological and evolutionary forces shaping microbial diversity in the human intestine. Cell 124:837-48

48. Ley RE, Turnbaugh PJ, Klein S, Gordon JI. 2006. Microbial ecology: human gut microbes associated with obesity. Nature 444:1022-23

49. Linz B, Balloux F, Moodley Y, Manica A, Liu H, et al. 2007. An African origin for the intimate association between humans and Helicobacter pylori. Nature 445:915-18

50. LoCascio RG, Desai P, Sela DA, Weimer B, Mills DA. 2010. Broad conservation of milk utilization genes in Bifidobacterium longum subsp. infantis as revealed by comparative genomic hybridization. Appl. Environ. Microbiol. 76:7373-81

51. Lozupone CA, Hamady M, Cantarel BL, Coutinho PM, Henrissat B, et al. 2008. The convergence of carbohydrate active gene repertoires in human gut microbes. Proc. Natl. Acad. Sci. USA 105:15076-81

52. Luca F, Perry G, Di Rienzo A. 2010. Evolutionary adaptations to dietary changes. Annu. Rev. Nutr. 30:291-314

53. Mandel AL, Peyrot des Gachons C, Plank KL, Alarcon S, Breslin PAS. 2010. Individual differences in AMY1 gene copy number, salivary alpha-amylase levels, and the perception of oral starch. PLoS ONE 5:e13352

54. Mantis NJ, Forbes SJ. 2010. Secretory IgA: Arresting microbial pathogens at epithelial borders. Immunol. Invest. 39:383-406

55. Martens EC, Chiang HC, Gordon JI. 2008. Mucosal glycan foraging enhances fitness and transmission of a saccharolytic human gut bacterial symbiont. Cell Host Microbe 4:447-57

56. Martinez I, Kim J, Duffy PR, Schlegel VL, Walter J. 2010. Resistant starches types 2 and 4 have differential effects on the composition of the fecal microbiota in human subjects. PLoS ONE 5:e15046

57. Mateos M, Castrezana SJ, Nankivell BJ, Estes AM, Markow TA, Moran NA. 2006. Heritable endosymbionts of Drosophila. Genetics 174:363-76

58. McFall-Ngai M. 2007. Adaptive immunity: care for the community. Nature 445:153

59. Moodley Y, Linz B, Yamaoka Y, Windsor HM, Breurec S, et al. 2009. The peopling of the Pacific from a bacterial perspective. Science 323:527-30

60. Moran NA, McCutcheon JP, Nakabachi A. 2008. Genomics and evolution of heritable bacterial symbionts. Annu. Rev. Genet. 42:165-90

61. Moran NA, Plague GR. 2004. Genomic changes following host restriction in bacteria. Curr. Opin. Genet. Dev. 14:627-33

62. Mottram DS, Wedzicha BL, Dodson AT. 2002. Food chemistry: Acrylamide is formed in the Maillard reaction. Nature 419:448-49

63. Normand P, Lapierre P, Tisa LS, Gogarten JP, Alloisio N, et al. 2007. Genome characteristics of facultatively symbiotic Frankia sp. strains reflect host range and host plant biogeography. Genome Res. 17:7-15

64. Nyholm S, McFall-Ngai M. 2004. The winnowing: establishing the squid-vibrio symbiosis. Nat. Rev. Microbiol. 2:632-42

65. Oh PL, Benson AK, Peterson DA, Patil PB, Moriyama EN, et al. 2010. Diversification of the gut symbiont Lactobacillus reuteri as a result of host-driven evolution. ISME 7. 4:377-87

66. Palmer C, Bik EM, Digiulio DB, Relman DA, Brown PO. 2007. Development of the human infant intestinal microbiota. PLoS Biol. 5:e177

67. Perry GH, Dominy NJ, Claw KG, Lee AS, Fiegler H, et al. 2007. Diet and the evolution of human amylase gene copy number variation. Nat. Genet. 39:1256-60

68. Peterson DA, McNulty NP, Guruge JL, Gordon JI. 2007. IgA response to symbiotic bacteria as a mediator of gut homeostasis. Cell Host Microbe 2:328-39

69. Putsep K, Axelsson LG, Boman A, Midtvedt T, Normark S, et al. 2000. Germ-free and colonized mice generate the same products from enteric prodefensins. 7. Biol. Chem. 275:40478-82

70. Qin J, Li R, Raes J, Arumugam M, Burgdorf K, et al. 2010. A human gut microbial gene catalogue established by metagenomic sequencing. Nature 464:59-65
51. Compares the genomes of gut microbes with those of other environments and shows patterns of convergent evolution.

52. Synthesizes human genetics and recent human evolution related to diet.

67. Documents higher amylase gene copy number in people with agricultural compared to hunter-gatherer backgrounds.

70. A large study of the metagenomic gene content of Western fecal microbial communities. 
72. Suggests that gut bacteria provide amino acids to the host, alleviating malnutrition in some hosts.
96. Provides an intriguing look into ancient gut microbiota and shows them to be remarkably similar to modern gut microbiomes.
71. Rainey PB, Travisano M. 1998. Adaptive radiation in a heterogeneous environment. Nature 394:69-72

72. Raj T, Dileep U, Vaz M, Fuller MF, Kurpad AV. 2008. Intestinal microbial contribution to metabolic leucine input in adult men. $\mathcal{F}$. Nutr. 138:2217-21

73. Ramsay A, Scott K, Martin J, Rincon M, Flint H. 2006. Cell-associated alpha-amylases of butyrateproducing Firmicute bacteria from the human colon. Microbiology 152:3281-90

74. Rawls JF, Mahowald MA, Ley RE, Gordon JI. 2006. Reciprocal gut microbiota transplants from zebrafish and mice to germ-free recipients reveal host habitat selection. Cell 127:423-33

75. Reyes A, Haynes M, Hanson N, Angly F, Heath A, et al. 2010. Viruses in the faecal microbiota of monozygotic twins and their mothers. Nature 466:334-38

76. Richerson PJ, Boyd R, Henrich J. 2010. Colloquium paper: gene-culture coevolution in the age of genomics. Proc. Natl. Acad. Sci. USA 107:8985-92

77. Robinson CJ, Bohannan BJ, Young VB. 2010. From structure to function: the ecology of host-associated microbial communities. Microbiol. Mol. Biol. Rev. 74:453-76

78. Rosenberg E, Sharon G, Atad I, Zilber-Rosenberg I. 2010. The evolution of animals and plants via symbiosis with microorganisms. Environ. Microbiol. Rep. 2:500-6

79. Salzman NH, Hung K, Haribhai D, Chu H, Karlsson-Sjöberg J, et al. 2010. Enteric defensins are essential regulators of intestinal microbial ecology. Nat. Immunol. 11:76-83

80. Savage DC. 1977. Microbial ecology of the gastrointestinal tract. Annu. Rev. Microbiol. 31:107-33

81. Scanlan P, Marchesi J. 2008. Micro-eukaryotic diversity of the human distal gut microbiota: qualitative assessment using culture-dependent and -independent analysis of faeces. ISME f. 2:1183-93

82. Sloan WT, Lunn M, Woodcock S, Head IM, Nee S, Curtis TP. 2006. Quantifying the roles of immigration and chance in shaping prokaryote community structure. Environ. Microbiol. 8:732-40

83. Solnick JV, Schauer DB. 2001. Emergence of diverse Helicobacter species in the pathogenesis of gastric and enterohepatic diseases. Clin. Microbiol. Rev. 14:59-97

84. Sonnenburg ED, Zheng H, Joglekar P, Higginbottom SK, Firbank SJ, et al. 2010. Specificity of polysaccharide use in intestinal Bacteroides species determines diet-induced microbiota alterations. Cell 141:124152

85. Sonnenburg JL, Xu J, Leip DD, Chen CH, Westover BP, et al. 2005. Glycan foraging in vivo by an intestine-adapted bacterial symbiont. Science 307:1955-59

86. Stadler R, Blank I, Varga N, Robert F, Hau J, et al. 2002. Food chemistry: acrylamide from Maillard reaction products. Nature 419:449-50

87. Stecher B, Hardt WD. 2008. The role of microbiota in infectious disease. Trends Microbiol. 16:107-14

88. Stevens CE, Hume I. 1998. Contributions of microbes in vertebrate gastrointestinal tract to production and conservation of nutrients. Physiol. Rev. 78:393-427

89. Stoel M, Jiang HQ, van Diemen CC, Bun JC, Dammers PM, et al. 2005. Restricted IgA repertoire in both B-1 and B-2 cell-derived gut plasmablasts. F. Immunol. 174:1046-54

90. Suzuki K, Meek B, Doi Y, Muramatsu M, Chiba T, et al. 2004. Aberrant expansion of segmented filamentous bacteria in IgA-deficient gut. Proc. Natl. Acad. Sci. USA 101:1981-86

91. Tamames J, Mira A. 2010. Horizontal gene transfer in prokaryotic microbiomes. In Metagenomics: Theory, Methods and Applications, ed. D Marco, pp. 39-53. Norwich, UK: Horizon Press

92. Tap J, Mondot S, Levenez F, Pelletier E, Caron C, et al. 2009. Towards the human intestinal microbiota phylogenetic core. Environ. Microbiol. 11:2574-84

93. Tasse L, Bercovici J, Pizzut-Serin S, Robe P, Tap J, et al. 2010. Functional metagenomics to mine the human gut microbiome for dietary fiber catabolic enzymes. Genome Res. 20:1605-12

94. Tenaillon O, Skurnik D, Picard B, Denamur E. 2010. The population genetics of commensal Escherichia coli. Nat. Rev. Microbiol. 8:207-17

95. Tishkoff S, Reed F, Ranciaro A, Voight B, Babbitt C, et al. 2006. Convergent adaptation of human lactase persistence in Africa and Europe. Nat. Genet. 39:31-40

96. Tito RY, Macmil S, Wiley G, Najar F, Cleeland L, et al. 2008. Phylotyping and functional analysis of two ancient human microbiomes. PLoS ONE 3:e3703

97. Tomb JF, White O, Kerlavage AR, Clayton RA, Sutton GG, et al. 1997. The complete genome sequence of the gastric pathogen Helicobacter pylori. Nature 388:539-47 
98. Torrallardona D, Harris CI, Fuller MF. 2003. Pigs' gastrointestinal microflora provide them with essential amino acids. F. Nutr. 133:1127-31

99. Touchon M, Hoede C, Tenaillon O, Barbe V, Baeriswyl S, et al. 2009. Organised genome dynamics in the Escherichia coli species results in highly diverse adaptive paths. PLoS Genet. 5:e1000344

100. Tuohy KM, Hinton DJS, Davies SJ, Crabbe MJC, Gibson GR, Ames JM. 2006. Metabolism of Maillard reaction products by the human gut microbiota-implications for health. Mol. Nutr. Food Res. 50:847-57

101. Turnbaugh PJ, Backhed F, Fulton L, Gordon J. 2008. Diet-induced obesity is linked to marked but reversible alterations in the mouse distal gut microbiome. Cell Host Microbe 3:213-23

102. Turnbaugh PJ, Hamady M, Yatsunenko T, Cantarel BL, Duncan A, et al. 2009. A core gut microbiome in obese and lean twins. Nature 457:480-84

103. Vaishnava S, Behrendt CL, Ismail AS, Eckmann L, Hooper LV. 2008. Paneth cells directly sense gut commensals and maintain homeostasis at the intestinal host-microbial interface. Proc. Natl. Acad. Sci. USA 105:20858-63

104. Walker AW, Ince J, Duncan SH, Webster LM, Holtrop G, et al. 2011. Dominant and diet-responsive groups of bacteria within the human colonic microbiota. ISME 7. 5:220-30

105. Walter J. 2008. Ecological role of lactobacilli in the gastrointestinal tract: implications for fundamental and biomedical research. Appl. Environ. Microbiol. 74:4985-96

106. Wang M, Ahrne S, Jeppsson B, Molin G. 2005. Comparison of bacterial diversity along the human intestinal tract by direct cloning and sequencing of 16S rRNA genes. FEMS Microbiol. Ecol. 54:219-31

107. Wang X, Heazlewood SP, Krause DO, Florin TH. 2003. Molecular characterization of the microbial species that colonize human ileal and colonic mucosa by using $16 \mathrm{~S}$ rDNA sequence analysis. 7 . Appl. Microbiol. 95:508-20

108. Willing BP, Dicksved J, Halfvarson J, Andersson AF, Lucio M, et al. 2010. A pyrosequencing study in twins shows that gastrointestinal microbial profiles vary with inflammatory bowel disease phenotypes. Gastroenterology 139:1844-54

109. Wobber V, Hare B, Wrangham R. 2008. Great apes prefer cooked food. F. Human Evol. 55:340-48

110. Woolnough J, Bird A, Monro J, Brennan C. 2010. The effect of a brief salivary $\alpha$-amylase exposure during chewing on subsequent in vitro starch digestion curve profiles. Int. 7. Mol. Sci. 11:2780-90

111. Zoetendal EG, von Wright A, Vilpponen-Salmela T, Ben-Amor K, Akkermans AD, de Vos WM. 2002. Mucosa-associated bacteria in the human gastrointestinal tract are uniformly distributed along the colon and differ from the community recovered from feces. Appl. Environ. Microbiol. 68:3401-7 